TITLE:

\title{
Model Order Reduction of an Induction Motor Using a Cauer Ladder Network
}

$\operatorname{AUTHOR}(\mathrm{S}):$

Matsuo, Tetsuji; Sugahara, Kengo; Kameari, Akihisa; Shindo, Yuji

\section{CITATION:}

Matsuo, Tetsuji ... [et al]. Model Order Reduction of an Induction Motor Using a Cauer Ladder Network. IEEE Transactions on Magnetics 2020, 56(3): 7514704.

ISSUE DATE:

2020-03

URL:

http://hdl.handle.net/2433/254199

RIGHT:

(c) 2020 IEEE. Personal use of this material is permitted. Permission from IEEE must be obtained for all other uses, in any current or future media, including reprinting/republishing this material for advertising or promotional purposes,

creating new collective works, for resale or redistribution to servers or lists, or reuse of any copyrighted component of this work in other works.; This is not the published version. Please cite only the published version.; この論文は出版社版

でありません。引用の際には出版社版をご確認ご利用ください。 


\title{
Model Order Reduction of an Induction Motor Using a Cauer Ladder Network
}

\author{
Tetsuji Matsuo ${ }^{1}$, Kengo Sugahara ${ }^{2}$, Akihisa Kameari ${ }^{3}$, and Yuji Shindo ${ }^{4}$ \\ ${ }^{1}$ Graduate School of Engineering, Kyoto University, Kyoto 615-8510, Japan \\ ${ }^{2}$ Faculty of Science and Engineering, Kindai University, Higashiosaka 577-8502, Japan \\ ${ }^{3}$ Science Solutions International Laboratory, Inc., Tokyo 153-0065, Japan \\ ${ }^{4}$ Kawasaki Heavy Industries, Ltd., Akashi 673-8666, Japan
}

\begin{abstract}
A method for motor model order reduction is developed using the Cauer ladder network. The analyzed domain is decomposed into stator and mover domains, where the multiport Cauer ladder network of each domain is constructed independently from the other. The two domains are connected through electromagnetic field modes at the boundary. The boundary condition is derived from the coordinate transformation. The reduced model accurately reconstructs the induction motor property affected by slot harmonics.
\end{abstract}

Index Terms - Cauer ladder network, domain decomposition, induction motor, multi-port model order reduction

\section{INTRODUCTION}

$\mathrm{M}$ ODEL order reduction (MOR) [1]-[5] has been intensively studied for the efficient description of the magnetic field. The Cauer ladder network (CLN) [5][6] is one of the promising MOR frameworks. The representation of the network is easy to combine with control theory and a circuit simulator. One emerging demand for MOR is the application to motor control for electric vehicles and robots. Studies have addressed motor MOR [2][3], realizing magnetostatic MOR depending on the rotation angle. However, motor MOR including the motional electromotive force (EMF) remains an open problem.

This study develops a systematic motor MOR method based on the multiport CLN method [7] and domain decomposition to handle the induced current in moving conductors for analysis of the induction motor. To confirm the basic concept of connecting decomposed domains reduced to multiport CLNs with spatial harmonic components, the two-dimensional eddy-current (EC) field in a linear motor is analyzed as a simple example.

\section{MultiPort CLN METHOD}

Vector potential $\boldsymbol{A}$ and electric field $\boldsymbol{E}$ are represented in finite element (FE) space as

$$
\boldsymbol{A}=\sum_{i} a_{i} \boldsymbol{w}_{i}^{1}, \quad \boldsymbol{E}=\sum_{i} e_{i} \boldsymbol{w}_{i}^{1},
$$

where $a_{i}$ and $e_{i}$ are line integrals of $\boldsymbol{A}$ and $\boldsymbol{E}$ on edge $i$ and $\boldsymbol{w}^{1}{ }_{i}$ is the edge element.

The EC field is described in FE space as

$$
\boldsymbol{C}^{\mathrm{T}} \boldsymbol{v} \boldsymbol{C a}=\boldsymbol{\sigma e}, \boldsymbol{C e}=-\boldsymbol{C} \frac{\mathrm{d} \boldsymbol{a}}{\mathrm{d} t},
$$

where $\boldsymbol{a}=\left[a_{1}, a_{2}, \ldots\right]^{\mathrm{T}}, \boldsymbol{e}=\left[e_{1}, e_{2}, \ldots\right]^{\mathrm{T}}, \boldsymbol{C}$ is the face-edge incidence matrix, and $\boldsymbol{C}^{\mathrm{T}} \mathbf{v} \boldsymbol{C}$ is the stiffness matrix for FE EC analysis. The conductivity matrix $\boldsymbol{\sigma}$ and reluctivity matrix $\mathbf{v}$ are given as

$$
\begin{aligned}
& \boldsymbol{v}=\left\{v_{k l}\right\}, v_{k l}=\int_{\Omega} \frac{1}{\mu} \boldsymbol{w}_{k}^{2} \cdot \boldsymbol{w}_{l}^{2} \mathrm{~d} \Omega, \\
& \boldsymbol{\sigma}=\left\{\sigma_{i j}\right\}, \sigma_{i j}=\int_{\Omega} \sigma \boldsymbol{w}_{i}^{1} \cdot \boldsymbol{w}_{j}^{1} \mathrm{~d} \Omega,
\end{aligned}
$$

where $\mu$ is the permeability and $\sigma$ is the conductivity; $i, j$ and $k$, $l$ are indices of edges and faces, respectively, while $\boldsymbol{w}^{2}{ }_{k}$ is the facial element of face $k$.

Let the number of ports be $M$. The multiport CLN [7] is constructed using the recurrence relation as

$$
\begin{aligned}
& \boldsymbol{C}^{\mathrm{T}} \boldsymbol{v} \boldsymbol{C}\left(\boldsymbol{a}_{2 n+1}-\boldsymbol{a}_{2 n-1}\right)=\boldsymbol{\sigma} \boldsymbol{e}_{2 n} \boldsymbol{R}_{2 n}, \\
& \boldsymbol{e}_{2 n+2}-\boldsymbol{e}_{2 n}=-\boldsymbol{a}_{2 n+1} \boldsymbol{L}_{2 n+1}{ }^{-1}, \\
& \boldsymbol{R}_{2 n}{ }^{-1}=\boldsymbol{e}_{2 n}^{\mathrm{T}} \boldsymbol{\sigma} \boldsymbol{e}_{2 n}, \boldsymbol{L}_{2 n-1}=\boldsymbol{a}_{2 n-1}^{\mathrm{T}} \boldsymbol{C}^{\mathrm{T}} \boldsymbol{v} \boldsymbol{C} \boldsymbol{a}_{2 n-1},
\end{aligned}
$$

where $\boldsymbol{R}_{2 n}$ and $\boldsymbol{L}_{2 n-1}$ are multiport resistance and inductance matrices while $\boldsymbol{e}_{2 n}=\left(\boldsymbol{e}_{1,2 n}, \ldots, \boldsymbol{e}_{M, 2 n}\right)$ and $\boldsymbol{a}_{2 n-1}=\left(\boldsymbol{a}_{1,2 n-1}, \ldots\right.$, $\left.\boldsymbol{a}_{M, 2 n-1}\right)$ are matrices of the $n$-th basis vectors. The $m$-th columns $\boldsymbol{e}_{m, 2 n}$ and $\boldsymbol{a}_{m, 2 n-1}$ are field basis vectors when the unit and zero power sources are fed to port $m$ and other ports, respectively. The basis vector matrices have the orthogonal property

$$
\boldsymbol{e}_{2 i}^{\mathrm{T}} \boldsymbol{\sigma} \boldsymbol{e}_{2 j}=\delta_{i j} \boldsymbol{R}_{2 i}{ }^{-1}, \boldsymbol{a}_{2 i+1}^{\mathrm{T}} \boldsymbol{C}^{\mathrm{T}} \boldsymbol{v} \boldsymbol{C} \boldsymbol{a}_{2 j+1}=\delta_{i j} \boldsymbol{L}_{2 i+1},
$$

where $\delta_{i j}$ is Kronecker's delta.

The electromagnetic fields are expanded using the basis vectors obtained above as

$$
\boldsymbol{a}=\sum_{n} \boldsymbol{a}_{2 n-1} \boldsymbol{I}_{2 n-1}, \quad \boldsymbol{e}=\sum_{n} \boldsymbol{e}_{2 n} \boldsymbol{V}_{2 n} .
$$

Therein, $\boldsymbol{I}_{2 n-1}$ and $\boldsymbol{V}_{2 n}$ are coefficient vectors, which are determined by the matrix Cauer network shown in Fig. 1. 


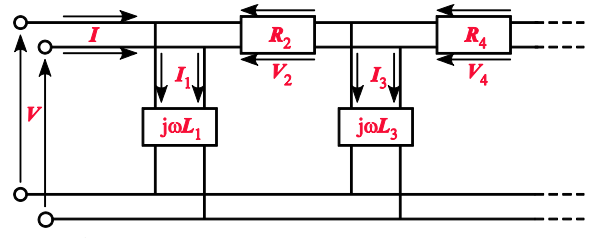

Fig. 1. Matrix Cauer form.

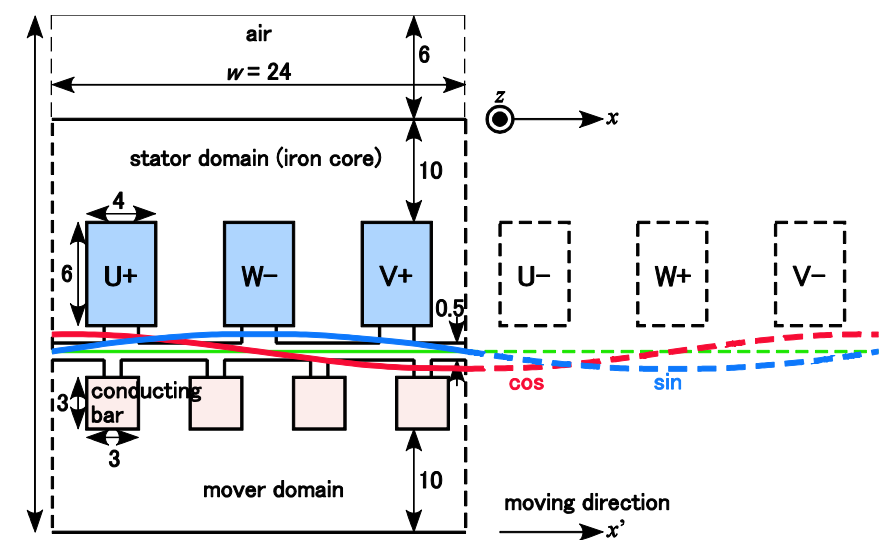

Fig. 2. Linear induction motor (unit: millimeter).

\section{MOTOR MOR}

\section{A. Connection of Stator and Mover Domains}

As a simple example, the linear induction motor shown in Fig. 2 is analyzed, where $x$ and $x$ ' are the stator-fixed and mover-fixed coordinates, respectively. The stator domain and mover domain are connected through the magnetic field $H_{x}$, electric field $E_{z}$, and/or the vector potential $A_{z}=-\int E_{\mathrm{z}} \mathrm{d} t$. The magnetic fields $H_{x}$ and $H_{x}{ }^{\prime}$ at the boundary surface on the stator and mover domain sides, respectively, are expanded into spatial harmonic components as

$$
\begin{aligned}
& H_{x}(x, t)=\sqrt{2} \sum_{\text {odd } m}\left[H_{\mathrm{c} m}(t) \cos (m k x)+H_{\mathrm{s} m}(t) \sin (m k x)\right], \\
& H_{x}^{\prime}\left(x^{\prime}, t^{\prime}\right)=\sqrt{2} \sum_{\mathrm{odd} m}\left[H_{\mathrm{c} m}^{\prime}\left(t^{\prime}\right) \cos \left(m k x^{\prime}\right)+H_{\mathrm{s} m}^{\prime}\left(t^{\prime}\right) \sin \left(m k x^{\prime}\right)\right]
\end{aligned}
$$

where $k=\pi / w$ and $w$ is half the spatial period [Fig. 1].

The harmonic components of the magnetic field are denoted

$$
\begin{aligned}
& \boldsymbol{I}=\left[H_{\mathrm{c} 1}, H_{\mathrm{s} 1}, \ldots, H_{\mathrm{c} 2 K-1}, H_{\mathrm{s} 2 K-1}\right], \\
& \boldsymbol{I}^{\prime}=\left[H^{\prime}{ }_{\mathrm{c} 1}, H^{\prime}{ }_{\mathrm{s} 1}, \ldots, H^{\prime}{ }_{\mathrm{c} 2 K-1}, H^{\prime}{ }_{\mathrm{s} 2 K-1}\right],
\end{aligned}
$$

where the components over the $\left(2 K^{-1}\right)$-th harmonics are truncated. The harmonic components of the electric field and vector potential are similarly denoted

$$
\begin{aligned}
& \boldsymbol{V}=w\left[E_{\mathrm{c} 1}, E_{\mathrm{s} 1}, \ldots, E_{\mathrm{c} 2 K-1}, E_{\mathrm{s} 2 K-1}\right], \\
& \boldsymbol{V}^{\prime}=w\left[E^{\prime}{ }_{\mathrm{c} 1}, E_{\mathrm{s} 1}, \ldots, E_{\mathrm{c} 2 K-1}^{\prime}, E_{\mathrm{s} 2 K-1}^{\prime}\right], \\
& \boldsymbol{\Phi}=w\left[A_{\mathrm{c} 1}, A_{\mathrm{s} 1}, \ldots, A_{\mathrm{c} 2 K-1}, A_{\mathrm{s} 2 K-1}\right], \\
& \boldsymbol{\Phi}^{\prime}=w\left[A^{\prime}{ }_{\mathrm{c} 1}, A^{\prime}{ }_{\mathrm{s} 1}, \ldots, A^{\prime}{ }_{\mathrm{c} 2 K-1}, A^{\prime}{ }_{\mathrm{s} 2 K-1}\right],
\end{aligned}
$$

where

$$
\boldsymbol{V}=-\frac{\mathrm{d} \boldsymbol{\Phi}}{\mathrm{d} t}, \boldsymbol{V}^{\prime}=-\frac{\mathrm{d} \boldsymbol{\Phi}^{\prime}}{\mathrm{d} t^{\prime}} .
$$

The coordinate relation is given as

$$
x^{\prime}=x-v t, t^{\prime}=t,
$$

where $v$ is the velocity of the mover domain. The boundary condition is obtained from (10)-(14) as

$$
I^{\prime}=\boldsymbol{T I}, \Phi^{\prime}=\boldsymbol{T} \Phi,
$$

where

$$
\begin{gathered}
\boldsymbol{T}=\operatorname{blockdiag}\left(\boldsymbol{T}_{1}, \ldots, \boldsymbol{T}_{2 K-1}\right), \\
\boldsymbol{T}_{m}=\left[\begin{array}{cc}
\cos (m k v t) & \sin (m k v t) \\
-\sin (m k v t) & \cos (m k v t)
\end{array}\right](m=1,3, \ldots) .
\end{gathered}
$$

The boundary condition between $\boldsymbol{V}$ and $\boldsymbol{V}^{\prime}$ is derived as follows. It follows from $B_{y}=-\partial A_{z} / \partial x$ that

$$
B_{y}(x, t)=\sqrt{2} \sum_{\mathrm{odd} m} m k\left[-A_{\mathrm{s} m}(t) \cos (m k x)+A_{\mathrm{c} m}(t) \sin (m k x)\right],
$$

where the harmonic components are denoted

$$
\boldsymbol{B}=k w\left[-A_{\mathrm{s} 1}, A_{\mathrm{c} 1}, \ldots,-\left(2 K^{-1}\right) A_{\mathrm{s} 2 K^{-1}},\left(2 K^{-1}\right) A_{\mathrm{c} 2 K^{-1}}\right] .
$$

Considering that $\partial / \partial t^{\prime}=\partial / \partial t+v \partial / \partial x$ and that (15) is independent of $x$,

$$
\boldsymbol{V}^{\prime}=-\frac{\mathrm{d} \boldsymbol{\Phi}^{\prime}}{\mathrm{d} t^{\prime}}=-\frac{\mathrm{d} \boldsymbol{T}}{\mathrm{d} t} \boldsymbol{\Phi}-\boldsymbol{T} \frac{\mathrm{d} \boldsymbol{\Phi}}{\mathrm{d} t}=\boldsymbol{T}(\boldsymbol{V}+v \boldsymbol{B})
$$

is obtained, where $v \boldsymbol{B}$ represents the motional EMF.

\section{B. Matrix Cauer Form Representation}

The three-phase currents and voltages are denoted

$$
\boldsymbol{I}_{\mathrm{S}}=\left[I_{\mathrm{U}}, I_{\mathrm{V}}, I_{\mathrm{W}}\right], \boldsymbol{V}_{\mathrm{S}}=\left[V_{\mathrm{U}}, V_{\mathrm{V}}, V_{\mathrm{W}}\right] .
$$

The multiport CLN procedure is executed independently in the stator and mover domains to derive the respective multiport transfer functions:

$$
\begin{aligned}
& \tilde{\boldsymbol{V}}=\tilde{\boldsymbol{Z}} \tilde{\boldsymbol{I}}, \boldsymbol{V}^{\prime}=\boldsymbol{Z}^{\prime} \boldsymbol{I}^{\prime}, \\
& \tilde{\boldsymbol{V}}=\left[\begin{array}{c}
\boldsymbol{V}_{\mathrm{S}} \\
\boldsymbol{V}
\end{array}\right], \tilde{\boldsymbol{I}}=\left[\begin{array}{c}
\boldsymbol{I}_{\mathrm{S}} \\
\boldsymbol{I}
\end{array}\right], \tilde{\boldsymbol{Z}}=\left[\begin{array}{cc}
\boldsymbol{Z}_{\mathrm{S}} & z^{\mathrm{T}} \\
\boldsymbol{z} & \boldsymbol{Z}
\end{array}\right] .
\end{aligned}
$$

These variables are used in both the frequency and time domains for the simplicity of notation. The CLN procedures for the stator and mover domains are started by setting

$$
\tilde{\boldsymbol{I}}=\boldsymbol{u}_{1}, \ldots, \boldsymbol{u}_{2 K+3}, \quad \boldsymbol{I}^{\prime}=\boldsymbol{u}_{1}, \ldots, \boldsymbol{u}_{2 K},
$$

where $\boldsymbol{u}_{m}$ is a unit vector whose $m$-th element is 1 and other elements are zero such that there is a unit source current or a unit magnetic-field component at the boundary. The latter is given by a Neumann boundary condition. Relations (22) and (23) are represented in Fig. 3(a) and (b), where $z$ is a mutual impedance matrix. Note that the CLN procedure for the mover domain yields the transfer function as in Fig. 3(c), where $\boldsymbol{I}^{\prime \prime}=$ $-I^{\prime}$ and $Z^{\prime \prime}=-Z^{\prime}$. The multiport transfer functions above are given in matrix continued fraction forms, which means that they are represented by matrix Cauer forms as shown in Fig. 1. Corresponding to Fig. 3(a), a sub-blockwise representation of the matrix Cauer form for the stator domain is shown in Fig. 4, where $\boldsymbol{m}_{2 n-1}$ and $\boldsymbol{r}_{2 n}(n=1,2, \ldots)$ are respectively mutual inductance and resistance matrices. A detailed representation of the matrix Cauer form for the mover domain is shown in Fig. 5 when $I^{\prime}=\left[H_{c 1}^{\prime}, H_{\text {s1 }}^{\prime}\right]$. These multiport networks for stator and mover domains are connected through relation (15). 


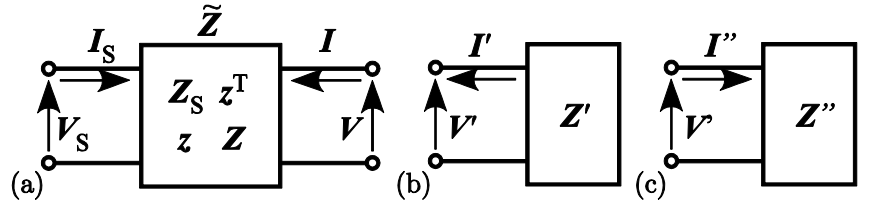

Fig. 3. Multiport transfer functions: (a) stator domain, (b) mover domain to be connected with stator domain, and (c) mover domain generated by the CLN.
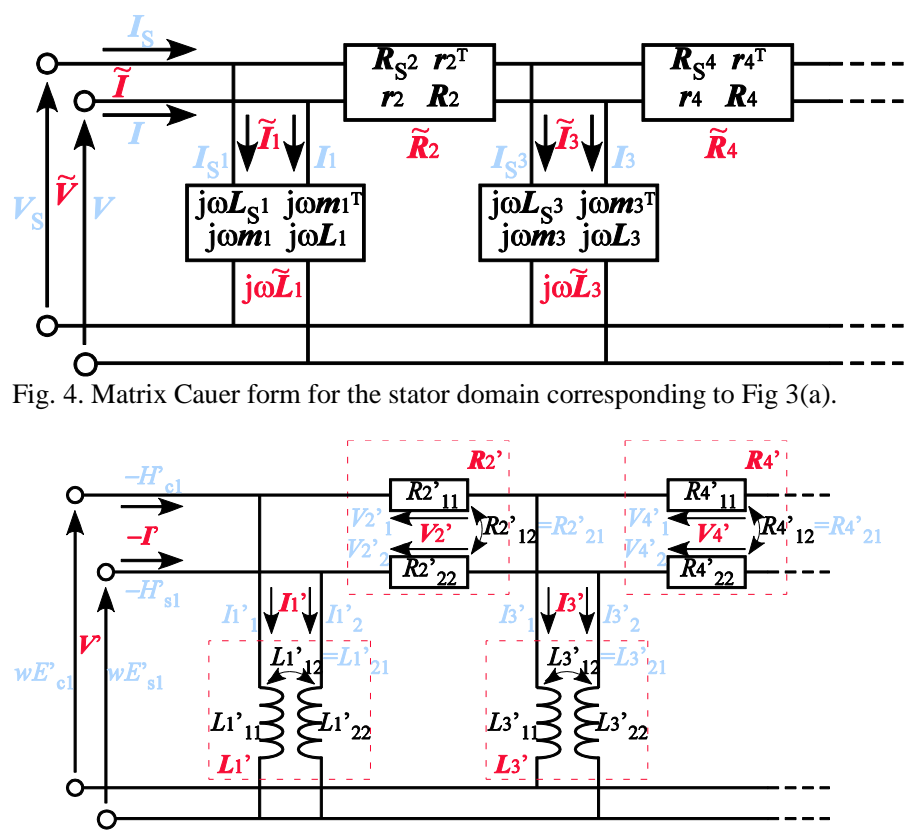

Fig. 5. Matrix Cauer form corresponding to Fig. 3(c) when $\boldsymbol{I}^{\prime}=\left[H_{\mathrm{c} 1}^{\prime}, H_{\mathrm{s} 1}^{\prime}\right]$.

\section{Homogenization of a Laminated Core}

A laminated iron core is usually used to reduce the eddycurrent loss. Even though it is possible for three-dimensional analysis to take account of the laminated structure, its computational cost is generally high because of the thinness of the sheet. Homogenization methods are often used to avoid mesh division on the sheet-thickness scale. The homogenization method in [8] is a useful method to be combined with FE analysis and can thus be built into the CLN method. The present paper focuses on motor MOR based on domain decomposition, combining simple zeroth-order homogenization with the CLN method.

Zeroth-order homogenization gives the constitutive relation between $\boldsymbol{B}$ and $\boldsymbol{H}$ as

$$
\boldsymbol{H}=v \boldsymbol{B}+\frac{\sigma d^{2}}{12} \frac{\mathrm{d} \boldsymbol{B}}{\mathrm{d} t}=v \operatorname{curl} \boldsymbol{A}+\frac{\sigma d^{2}}{12} \operatorname{curl} \frac{\mathrm{d} \boldsymbol{A}}{\mathrm{d} t},
$$

where $d$ is the sheet thickness. Using (25), governing equation (2) is rewritten as

$$
\boldsymbol{C}^{\mathrm{T}} \boldsymbol{v} \boldsymbol{C a}=-\boldsymbol{\sigma}^{\prime} \frac{\mathrm{d} \boldsymbol{a}}{\mathrm{d} t},
$$

where

$$
\begin{aligned}
& \boldsymbol{\sigma}^{\prime}=\boldsymbol{\sigma}+\boldsymbol{C}^{\mathrm{T}} \boldsymbol{v}^{\prime} \boldsymbol{C}, \\
& \boldsymbol{v}^{\prime}=\left\{v_{k l}^{\prime}\right\}, \quad v_{k l}^{\prime}=\int_{\Omega} \frac{\sigma d^{2}}{12} \boldsymbol{w}_{k}^{2} \cdot \boldsymbol{w}_{l}^{2} \mathrm{~d} \Omega .
\end{aligned}
$$

Replacing $\boldsymbol{\sigma}$ with $\boldsymbol{\sigma}^{\prime}$, the multiport CLN procedure is executed as in section II. The combination of higher-order homogenization with the CLN method will be reported in future work.

\section{Circuit Equation}

The circuit equation for the stator domain is given as

$$
\begin{aligned}
& \tilde{\boldsymbol{L}}_{2 n-1} \frac{\mathrm{d} \tilde{\boldsymbol{I}}_{2 n-1}}{\mathrm{~d} t}-\tilde{\boldsymbol{L}}_{2 n+1} \frac{\mathrm{d} \tilde{\boldsymbol{I}}_{2 n+1}}{\mathrm{~d} t}, \\
= & \tilde{\boldsymbol{R}}_{2 n}\left(\tilde{\boldsymbol{I}}-\tilde{\boldsymbol{I}}_{1}-\tilde{\boldsymbol{I}}_{3}-\ldots-\tilde{\boldsymbol{I}}_{2 n-1}\right) \\
\tilde{\boldsymbol{I}}= & \tilde{\boldsymbol{I}}_{1}+\tilde{\boldsymbol{I}}_{3}+\ldots+\tilde{\boldsymbol{I}}_{2 N_{\mathrm{s}}-1}+\tilde{\boldsymbol{R}}_{2 N_{\mathrm{s}}}{ }^{-1} \tilde{\boldsymbol{L}}_{2 N_{\mathrm{s}}-1} \frac{\mathrm{d} \tilde{\boldsymbol{I}}_{2 N_{\mathrm{s}}-1}}{\mathrm{~d} t},
\end{aligned}
$$

where the circuit is terminated by the $N_{\mathrm{s}}$-th resistor matrix $\boldsymbol{R}_{2 N \mathrm{~s}}$. Similarly, the circuit equation for the mover domain is given as

$$
\begin{aligned}
& \boldsymbol{L}_{2 n-1}^{\prime} \frac{\mathrm{d} \boldsymbol{I}_{2 n-1}^{\prime}}{\mathrm{d} t}-\boldsymbol{L}_{2 n+1}^{\prime} \frac{\mathrm{d} \boldsymbol{I}_{2 n+1}^{\prime}}{\mathrm{d} t} \\
& =\boldsymbol{R}_{2 n}^{\prime}\left(-\boldsymbol{I}^{\prime}-\boldsymbol{I}_{1}^{\prime}-\boldsymbol{I}_{3}^{\prime}-\ldots-\boldsymbol{I}_{2 n-1}^{\prime}\right) \\
& \boldsymbol{I}^{\prime}=-\boldsymbol{I}_{1}^{\prime}-\boldsymbol{I}_{3}^{\prime}-\ldots-\boldsymbol{I}_{2 N_{\mathrm{m}-1}}^{\prime}-\boldsymbol{R}_{2 N_{\mathrm{m}}}^{\prime}{ }^{-1} \boldsymbol{L}_{2 N_{\mathrm{m}}-1}^{\prime} \frac{\mathrm{d} \boldsymbol{I}_{2 N_{\mathrm{m}}-1}^{\prime}}{\mathrm{d} t},
\end{aligned}
$$

where the circuit is terminated by the $N_{\mathrm{m}}$-th resistor matrix $\boldsymbol{R}_{2 \mathrm{Nm}}$. To connect the two domains, (15) is written as

$$
\boldsymbol{I}^{\prime}(t)=\boldsymbol{T}(t) \boldsymbol{I}(t), \boldsymbol{L}_{1}{ }^{\prime} \boldsymbol{I}_{1}{ }^{\prime}(t)=\boldsymbol{T}(t)\left[\boldsymbol{m}_{1} \boldsymbol{I}_{\mathrm{S} 1}(t)+\boldsymbol{L}_{1} \boldsymbol{I}_{1}(t)\right],
$$

where $\boldsymbol{L}_{1}$ and $\boldsymbol{I}_{1}$ are the first inductance matrix and its current vector in the stator CLN [Fig. 4] while $\boldsymbol{L}_{1}{ }^{\prime}$ and $\boldsymbol{I}_{1}{ }^{\prime}$ are the first inductance matrix and its current vector in the mover CLN [Fig. 5].

The air-gap power $P_{2}$ transferred to the mover domain is given by $P_{2}=V^{\mathrm{T}} \boldsymbol{I}$ which corresponds to the Poynting vector. The thrust force $F_{x}=\int_{0}{ }^{w} H_{x} B_{y} \mathrm{~d} x$ at the boundary is obtained from (11) and (19) as

$$
F_{x}=\boldsymbol{I}^{\mathrm{T}} \boldsymbol{B} .
$$

\section{NUMERICAL RESULTS}

Sinusoidal three-phase current $\boldsymbol{I}_{\mathrm{S}}$ with an amplitude of 1 AT is fed to the linear induction motor [Fig. 2]. The relative permeability of the iron core is 1000 and the conductivity of the mover bar is $3 \times 10^{7} \mathrm{~S} / \mathrm{m}$. The sheet thickness and conductivity of the laminated core are respectively $0.35 \mathrm{~mm}$ and $2 \times 10^{6} \mathrm{~S} / \mathrm{m}$. The resistance of stator windings is not included in the stator-side CLN and can be inserted between the power source and stator-side CLN. Fig. 6 shows the magnetic flux $\int V_{\mathrm{V}} \mathrm{d} t$ and voltage $V_{\mathrm{U}}$ at $50 \mathrm{~Hz}$ with slip $s=1-$ $k v / \omega=0.25$, where root mean squared errors from the FE EC analysis are shown in parentheses. The slot harmonic components are exactly converted to the time harmonics by including up to the 17th spatial harmonics. Fig. 7 shows the impedance $R+\mathrm{j} \omega L$ seen from one of the three-phase ports whereas Fig. 8 shows the speed dependence of the air-gap power and thrust force. Differences of computed values from those obtained by the FE EC analysis are indicated in parentheses. When only the fundamental components are considered, the thrust force is zero when $s=0$. However, the thrust force is negative because of the higher harmonic 
components, as is accurately reproduced by the reduced model. On the basis of expansion (9), the vector potential and magnetic flux lines are reconstructed as in Fig. 9(a), where $s=$ 0.25. A comparison with the FE EC analysis [Fig. 9(b)] shows that the vector potential is accurately reconstructed. In the numerical experiment above with up to the 17-th harmonics, the construction of stator and mover CLNs with three stages takes $2.59 \mathrm{~ms}$ using a personal computer. The time-dependent computation of 144 steps $\times 25$ periods requires 0.73 and 145 $\mathrm{ms}$ for the reduced network computation and FE EC analysis, respectively. Consequently, in this case, the speed-up ratios for the total computation and time-dependent analysis are respectively 43.7 and 198.

\section{CONCLUSION}

A method for motor MOR was developed. The domain decomposition into stator and mover domains allows multiport CLNs to be constructed independently. The two domains are connected through electromagnetic field modes with the boundary condition derived from the coordinate transformation. The reduced model accurately reconstructs the induction motor property affected by slot harmonics. The application of the developed method to rotational machines is straightforward. The non-linearization of CLN method [9] will be combined for practical motor analyses.

\section{ACKNOWLEDGMENT}

We thank Glenn Pennycook, MSc, from Edanz Group (www.edanzediting.com/ac) for editing a draft of this manuscript.

\section{REFERENCES}

[1] P. Feldmann and R. W. Freund, "Efficient linear circuit analysis by Padé approximation via the Lanczos process," IEEE Trans. Comput.-Aided Design Integr. Circuits Syst., vol. 14, no. 5, pp. 639-649, May 1995.

[2] T. Henneron and S. Clénet, "Model order reduction applied to the numerical study of electrical motor based on POD method taking into account rotation movement," Int. J. Numer. Model., vol. 27, pp. 485-494, Feb. 2014.

[3] T. Shimotani, Y. Sato, T. Sato, and H. Igarashi, "Fast finite-element analysis of motors using block model order reduction," IEEE Trans. Magn., vol. 52, 7207004, Mar 2016

[4] Y. Sato and H. Igarashi, "Generation of equivalent circuit from finiteelement model using model order reduction," IEEE Trans. Magn., vol. 52, 1100304, Mar. 2016.

[5] A. Kameari, H. Ebrahimi, K. Sugahara, Y. Shindo and T. Matsuo, "Cauer ladder network representation of eddy-current fields for model order reduction using finite element method," IEEE Trans. Magn., vol. 54, no. 3, 7201804, Mar. 2018.

[6] T. Matsuo, A. Kameari, K. Sugahara, and Y. Shindo, "Matrix formulation of the Cauer ladder network method for efficient eddycurrent analysis," IEEE Trans. Magn., vol. 54, 7205805, Nov. 2018.

[7] T. Matsuo, T. Fujiwara, K. Kuriyama, K. Sugahara, A. Kameari, T. Tokumasu, Y. Shindo, "Multi-port model order reduction using matrix Cauer ladder network," IEEE Trans. Magn. (accepted for publication).

[8] J. Gyselinck, P. Dular, "A time-domain homogenization technique for laminated iron cores in 3-D finite-element models," IEEE Trans. Magn., vol. 40, pp. 856-859, Mar. 2004.

[9] H. Eskandari, T. Matsuo, "Cauer ladder network representation of nonlinear eddy-current field using first order approximation," IEEE Trans. Magn. (accepted for publication).
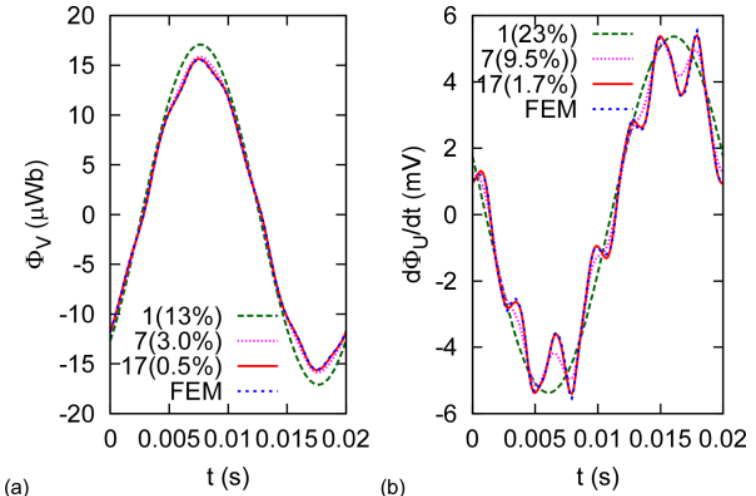

Fig. 6. Waveforms: (a) magnetic flux $\Phi_{\mathrm{V}}$ and (b) voltage $\mathrm{d} \Phi_{\mathrm{U}} / \mathrm{d} t$ where root mean squared errors from FE EC analysis are indicated in parentheses.
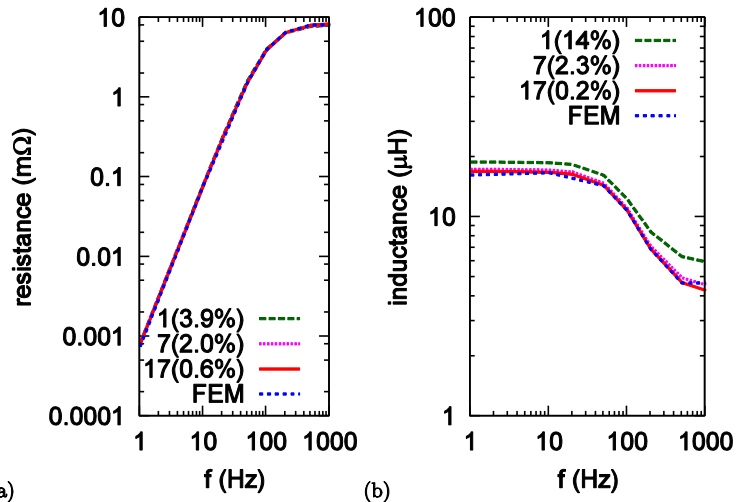

Fig. 7. Frequency dependence of the input impedance: (a) resistive and (b) inductive components. Discrepancies at $100 \mathrm{~Hz}$ are shown in parentheses.

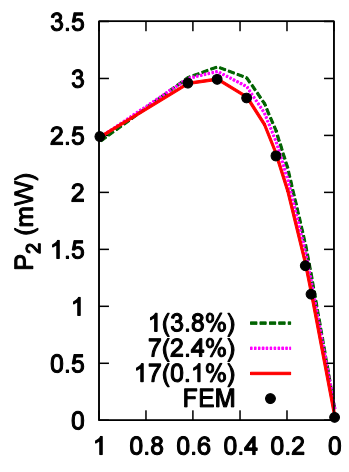

(a)

slip

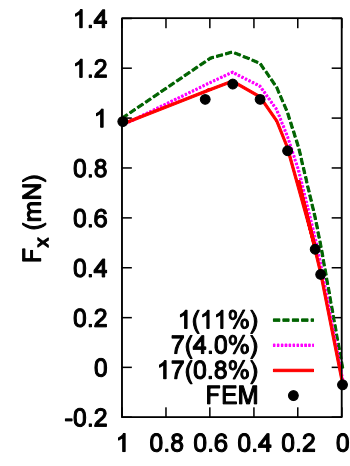

(b)

slip

Fig. 8. Speed dependence of (a) the air-gap power and (b) the thrust force. Discrepancies from FE EC analysis at $s=0.5$ are indicated in parentheses.

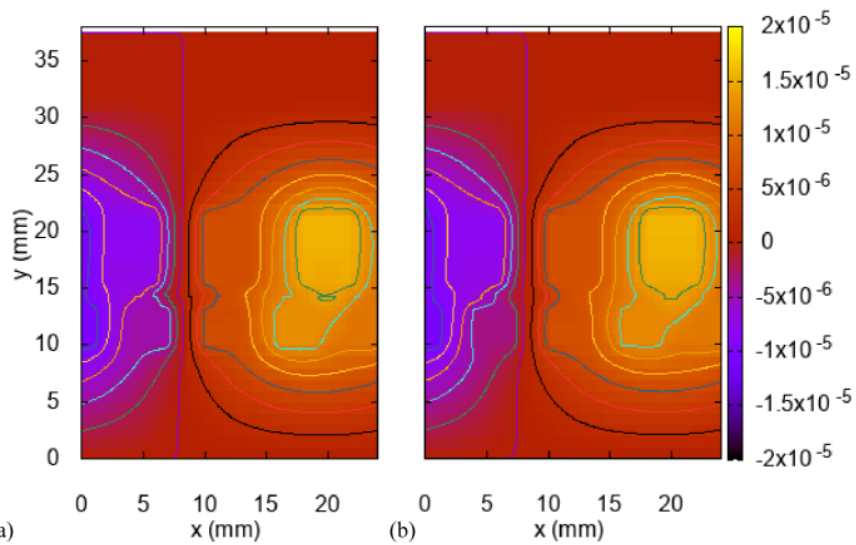

Fig. 9. Snapshots of the vector potential distribution and magnetic flux lines given by (a) motor MOR and (b) FE EC analysis. 